\title{
A SEMILINEAR TRANSPORT EQUATION WITH DELAYS
}

\author{
JANET DYSON, ROSANNA VILLELLA-BRESSAN, and GLENN F. WEBB
}

\author{
Received 26 November 2002
}

\begin{abstract}
An analysis is provided for a model of the blood production system based on a cell population structured by a continuous variable corresponding to the maturity of individual cells. Cell maturity is viewed as an indicator of increasing morphological development, ranging from the most primitive stem cells to the most mature differentiated cells. The analysis distinguishes two fundamental behaviors based on the nature of the initial state of the system: the first is a normal production of cells, when the initial state contains a sufficient supply of the least mature cells; the second is an abnormal production, when the initial state is deficient in the population of the least mature cells.
\end{abstract}

2000 Mathematics Subject Classification: 35R10, 92D25.

1. Introduction. In this paper, we investigate a singular transport equation which arises as a model of the blood production system. Similar models have been investigated in $[1,4,5,6,7,8,9]$. In the model we study here, which was developed by Rey and Mackey in [10], the blood production system is viewed as a population of cells with individual cells distinguished by a maturity variable $x$. The maturity value $x$ ranges from 0 to 1 , with maturity level corresponding to an increasingly developed level of cell type. In this model cells of all maturity values are capable of dividing. By successive divisions cell lines progress through increasingly mature cell types, which finally enter into the blood circulation. The division process is not modeled directly, but is accounted for by a nonlinear term which allows for a feedback control of cell production dependent upon the total cell population size.

We model these biological processes by a nonlinear partial differential equation for the cell population density which has cell maturity and time as independent variables. The equation of the model is a simplification of a more general model of cell population transport based upon cell maturity, time, and cell age. An idealized reduction of the 2-variable maturity-age structured model to the 1-structure variable maturity model yields an equation with a delay in the time variable and a scaling of the maturity variable (see [10]). The resulting equation has a novel character which is amenable to the theory of abstract functional differential equations. In [2], the authors made an investigation of this equation for the case that the nonlinear production term has a logistic form. 
Here we generalize our earlier results to the case that the nonlinear production term has a more general form, that is, we consider the equation

$$
\begin{gathered}
u_{t}(x, t)+(g(x) u(x, t))_{x}=f(u(\alpha x, t-\tau)), \quad 0 \leq x \leq 1, t>0, \\
u(x, t)=\phi(x, t) \in Y, \quad 0 \leq x \leq 1,-\tau \leq t \leq 0,
\end{gathered}
$$

where the delays $\alpha$ and $\tau$ are such that $0<\alpha<1$ and $\tau \geq 0$ and $Y$ is the space of real continuous functions on $[0,1] \times[-\tau, 0]$.

Our main goal is to distinguish abnormal behavior dependent upon properties of the linear maturation process $(g(x) u(x, t))_{x}$, the nonlinear proliferation process $f(u(\alpha x, t-\tau))$, and the initial values for the equation $\phi(x, t)$. If there is a sufficient supply of the least mature cell types (the initial maturity density of blood cells is strictly positive at the maturity value $x=0$ ), then there is a normal production of blood cells. If there is an insufficient supply of the least mature cell types (the initial maturity density of cells is 0 at the maturity value $x=0$ ), then there is an abnormal production of blood cells. In this situation we show that either every initial value is unstable or every initial value leads to extinction over time. Although the model contains many simplifying assumptions for the complex biological processes, it describes a destabilization of a process that occurs when its starting value is defective. It is believed that the pathology of aplastic anemia, a disease of the blood production system, is due to injury or destruction of a common pluripotential stem cell, which affects all subsequent cell populations (see [3]).

2. The semigroup generated by $-(g(x) u(x))^{\prime}$. In this section, we study the semigroup generated by the operator $(A u)(x)=-(g(x) u(x))^{\prime}$, where $g$ satisfies the following hypothesis:

$\left(\mathrm{H}_{g}\right) g \in C^{1}[0,1], g(0)=0$ and $g(x)>0$ for $0<x \leq 1$, and $\int_{0}^{1}(d s / g(s))=\infty$. The function $g$ in hypothesis $\left(\mathrm{H}_{g}\right)$ controls the maturation rate of individual cells, which all mature in exactly the same way. The time required for a cell to mature from $x_{1}$ to $x_{2}$ is given by $\int_{x_{1}}^{x_{2}}(d s / g(s))$, where $0 \leq x_{1}<x_{2} \leq 1$.

We prove the following proposition.

Proposition 2.1. Suppose that $g$ satisfies $\left(H_{g}\right)$. Then the operator $A$, defined by

$$
\begin{gathered}
D_{A}=\{u \in C[0,1], u \text { is differentiable on }(0,1], \\
\left.u^{\prime} \in C(0,1], \lim _{x \rightarrow 0} g(x) u^{\prime}(x)=0\right\}, \\
(A u)(x)=-(g(x) u(x))^{\prime}, \quad \text { if } 0<x \leq 1, \quad(A u)(0)=-g^{\prime}(0) u(0),
\end{gathered}
$$

is the infinitesimal generator of the semigroup

$$
(S(t) \psi)(x)=\exp \left(-\int_{0}^{t} g^{\prime}\left(h^{-1}\left(h(x) e^{-s}\right)\right) d s\right) \psi\left(h^{-1}\left(h(x) e^{-t}\right)\right),
$$

where $h(x)=\exp \left(\int_{1}^{x}(1 / g(s)) d s\right)$. 
Before proving Proposition 2.1, we first consider the operator $B: D_{B} \subset$ $C[0,1] \rightarrow C[0,1]$ defined by

$$
\begin{gathered}
D_{B}=\{u \in C[0,1], u \text { is differentiable on }(0,1], \\
\left.u^{\prime} \in C(0,1], \lim _{x \rightarrow 0} g(x) u^{\prime}(x)=0\right\}, \\
(B u)(x)=-g(x) u^{\prime}(x), \quad \text { if } 0<x \leq 1, \quad(B u)(0)=0 .
\end{gathered}
$$

We prove the following proposition.

Proposition 2.2. Suppose that $g$ satisfies $\left(H_{g}\right)$. Then the operator $B$ is the infinitesimal generator of a linear contraction semigroup on $C[0,1]$.

Proof. We have to prove that, given $w \in C[0,1]$ and $\lambda>0$, there exists a unique $u \in D_{B}$ such that

$$
(I-\lambda B) u=w
$$

and that $|u|_{\infty} \leq|w|_{\infty}$, where $|u|_{\infty}=\sup _{0 \leq x \leq 1}|u(x)|$.

To solve (2.4) we have to find $u \in C[0,1]$ which is a solution of the differential equation

$$
u(x)+\lambda g(x) u^{\prime}(x)=w(x), \quad 0<x \leq 1,
$$

and satisfies $u(0)=w(0)$.

The solutions of (2.5) are the functions

$u(x)=\exp \left(-\int_{1}^{x} \frac{1}{\lambda g(s)} d s\right)\left(k+\int_{1}^{x} \frac{w(t)}{\lambda g(t)} \exp \left(\int_{1}^{t} \frac{1}{\lambda g(\sigma)} d \sigma\right) d t\right), \quad 0<x \leq 1$,

for each $k \in \mathbb{R}$. Set

$$
h_{\lambda}(x)=\exp \left(\int_{1}^{x} \frac{d s}{\lambda g(s)}\right), \quad h_{\lambda}(0)=0,
$$

where $h_{\lambda}$ is continuous on $[0,1]$ and $h_{\lambda}^{\prime}(x)=h_{\lambda}(x) / \lambda g(x)$ on $(0,1]$. It follows that $\int_{1}^{x}\left(h_{\lambda}(t) / \lambda g(t)\right) d t=\int_{1}^{x} h_{\lambda}^{\prime}(t) d t=h_{\lambda}(x)-h_{\lambda}(1) \rightarrow-h_{\lambda}(1)$ as $x \rightarrow 0$, and so $h_{\lambda}(x) / \lambda g(x) \in L^{1}(0,1)$ and so is $w(x)\left(h_{\lambda}(x) / \lambda g(x)\right)$. As $h_{\lambda}(0)=0, u(x)$ given by (2.6), that is,

$$
u(x)=\frac{1}{h_{\lambda}(x)}\left(k+\int_{1}^{x} \frac{w(t)}{\lambda g(t)} h_{\lambda}(t) d t\right), \quad 0<x \leq 1,
$$

is bounded only if $k=\int_{1}^{0}(w(t) / \lambda g(t)) h_{\lambda}(t) d t$, so the only possible solution is

$$
u(x)=\frac{1}{h_{\lambda}(x)} \int_{0}^{x} \frac{w(t)}{\lambda g(t)} h_{\lambda}(t) d t, \quad 0<x \leq 1, \quad u(0)=w(0) .
$$

It is easy to check that in fact this function belongs to $D_{B}$. 
Also

$$
|u(x)| \leq \frac{|w|_{\infty}}{h_{\lambda}(x)} \int_{0}^{x} \frac{h_{\lambda}(t)}{\lambda g(t)} d t=|w|_{\infty}, \quad 0<x \leq 1, \quad|u(0)|=|w(0)|,
$$

and so $|u|_{\infty} \leq|w|_{\infty}$, as required.

Finally note that $\bar{D}_{B}=C[0,1]$.

If $\psi \in D_{B}$, then

$$
\lim _{t \rightarrow 0^{+}} \frac{\psi\left(h^{-1}\left(h(x) e^{-t}\right)\right)-\psi(x)}{t}=-g(x) \psi^{\prime}(x),
$$

hence the semigroup generated by $B$ is

$$
(T(t) \psi)(x)=\psi\left(h^{-1}\left(h(x) e^{-t}\right)\right),
$$

where $h(x)=\exp \left(\int_{1}^{x}(1 / g(s)) d s\right)$.

Proof of Proposition 2.1. Since $A$ is a bounded perturbation of $B, A$ is the infinitesimal generator of a semigroup on $C[0,1]$. To prove that this semigroup is given by (2.2), observe that for $\psi \in D(B)$, the solution $w(x, t)$ of the problem

$$
\frac{\partial}{\partial t}(g(x) w(x, t))=-g(x) \frac{\partial}{\partial x}(g(x) w(x, t)), \quad w(x, 0)=\psi(x),
$$

is

$$
w(x, t)=\frac{(T(t)(g \psi))(x)}{g(x)}=\frac{g\left(h^{-1}\left(h(x) e^{-t}\right)\right) \psi\left(h^{-1}\left(h(x) e^{-t}\right)\right)}{g(x)}
$$

for $x \in(0,1]$. To verify that (2.2) agrees with $w(x, t)$, it suffices to show that

$$
\exp \left(-\int_{0}^{t} g^{\prime}\left(h^{-1}\left(h(x) e^{-s}\right)\right) d s\right)=\frac{g\left(h^{-1}\left(h(x) e^{-t}\right)\right)}{g(x)}, \quad x \in(0,1],
$$

or equivalently,

$$
\log (g(x))-\int_{0}^{t} g^{\prime}\left(h^{-1}\left(h(x) e^{-s}\right)\right) d s=\log \left(g\left(h^{-1}\left(h(x) e^{-t}\right)\right)\right), \quad x \in(0,1] .
$$

The two sides of (2.16) agree at $t=0$ and differentiation of both sides with respect to $t$ yields

$$
g^{\prime}\left(h^{-1}\left(h(x) e^{-t}\right)\right)=\frac{g^{\prime}\left(h^{-1}\left(h(x) e^{-t}\right)\right) h^{-1^{\prime}}\left(h(x) e^{-t}\right) h(x) e^{-t}}{g\left(h^{-1}\left(h(x) e^{-t}\right)\right)} .
$$

Then (2.15) follows from the formula for $h$ since

$$
\left(h^{-1}\right)^{\prime}(u)=\frac{1}{\left(h^{-1}\right)^{\prime}\left(h^{-1}(u)\right)}=\frac{g\left(h^{-1}(u)\right)}{h\left(h^{-1}(u)\right)} .
$$


To verify that formula (2.2) satisfies the semigroup property, observe that

$$
\begin{aligned}
(S(t) S(s) \psi)(x)= & \frac{g\left(h^{-1}\left(h(x) e^{-t}\right)\right)}{g(x)}(S(s) \psi)\left(h^{-1}\left(h(x) e^{-t}\right)\right) \\
= & \frac{g\left(h^{-1}\left(h(x) e^{-t}\right)\right)}{g(x)} \frac{g\left(h^{-1}\left(h\left(h^{-1}\left(h(x) e^{-t}\right)\right) e^{-s}\right)\right)}{g\left(h^{-1}\left(h(x) e^{-t}\right)\right)} \\
& \times \psi\left(h^{-1}\left(h\left(h^{-1}\left(h(x) e^{-t}\right)\right) e^{-s}\right)\right) \\
= & \frac{g\left(h^{-1}\left(h(x) e^{-(t+s)}\right)\right)}{g(x)} \psi\left(h^{-1}\left(h(x) e^{-(t+s)}\right)\right) \\
= & (S(t+s) \psi)(x) .
\end{aligned}
$$

We note that in [2] the case $g(x)=x$ was considered.

3. Existence and uniqueness of solutions. Consider the equation

$$
\begin{gathered}
u_{t}(x, t)+(g(x) u(x, t))_{x}=f(u(\alpha x, t-\tau)), \quad 0 \leq x \leq 1, t>0, \\
u(x, t)=\phi(x, t) \in Y, \quad 0 \leq x \leq 1, \quad-\tau \leq t \leq 0, \tau>0, \\
u(x, 0)=\phi(x) \in X, \quad \tau=0,
\end{gathered}
$$

where the delays $\alpha$ and $\tau$ are such that $0<\alpha<1$ and $\tau \geq 0$. If $\tau>0$, then $Y$ is the space of real continuous functions on $[0,1] \times[-\tau, 0]$ with norm $\|\varphi\|=$ $\sup _{0 \leq x \leq 1,-\tau \leq t \leq 0}|\varphi(x, t)|$; so $Y=C([0,1] \times[-\tau, 0])=C([-\tau, 0] ; C[0,1])$. If $\tau=$ 0 , then $X=C[0,1]$ with norm $|\phi|_{\infty}$.

We suppose that $g$ satisfies $\left(\mathrm{H}_{g}\right)$, that is, $g \in C^{1}[0,1], g(0)=0$ and $g(x)>0$ for $0<x \leq 1$, and $\int_{0}^{1}(d s / g(s))=\infty$, and that $f: \mathbb{R} \rightarrow \mathbb{R}$ is continuous. The function $f$ incorporates the proliferation process of the population through cell division and cell mortality. For the existence and uniqueness of solutions, we require continuity and, in certain cases, differentiability conditions on $f$. For the asymptotic behavior of the solutions, we will require differentiability conditions on $f$ related to differentiability conditions on $g$.

From the results of Section 1, it follows that the mild or integrated version of (3.1) is the integral equation

$$
\begin{gathered}
u(x, t)=\phi\left(h^{-1}\left(h(x) e^{-t}\right), 0\right) \exp \left(-\int_{0}^{t} g^{\prime}\left(h^{-1}\left(h(x) e^{-\xi}\right)\right) d \xi\right) \\
+\int_{0}^{t} f\left(u\left(\alpha h^{-1}\left(h(x) e^{-(t-\sigma)}\right), \sigma-\tau\right)\right) \\
\times \exp \left(-\int_{0}^{t-\sigma} g^{\prime}\left(h^{-1}\left(h(x) e^{-\xi}\right)\right) d \xi\right) d \sigma, \quad 0 \leq x \leq 1, t>0, \\
u(x, t)=\phi(x, t) \in Y, \quad 0 \leq x \leq 1, \quad-\tau \leq t \leq 0, \tau>0, \\
u(x, 0)=\phi(x) \in X, \quad \tau=0 .
\end{gathered}
$$

In this section, we study existence and uniqueness of the solutions of (3.2). If $\tau>0$, it is easily seen, using the method of steps, that (3.2) has a continuous 
solution for each continuous initial datum and that this solution is unique. We prove in Proposition 3.1 a specific uniqueness result that will be useful in the instability analysis of Section 5. This result says that if two initial data agree for small values of the maturity variable $x$, then their corresponding solutions will ultimately agree for sufficiently large $t$ for all maturity values $x$.

Proposition 3.1. Let $\tau \geq 0$. Suppose that $u_{1}(x, t)$ and $u_{2}(x, t)$ are solutions of (3.2) with initial data $\varphi_{1}$ and $\varphi_{2}$, respectively. Suppose that there exists $b$, $0<b \leq 1$, such that

$$
\varphi_{1}(x, t)=\varphi_{2}(x, t) \quad \text { for } x \in[0, b], t \in[-\tau, 0], \text { if } \tau>0,
$$

or

$$
\varphi_{1}(x)=\varphi_{2}(x) \quad \text { for } x \in[0, b], \text { if } \tau=0 .
$$

Then there exists $\bar{t}$ such that $u_{1}(x, t)=u_{2}(x, t)$ for $x \in[0,1]$ and $t \geq \bar{t}$, where $\bar{t}$ can be chosen to be $-\log h(b)+\log b / \log \alpha$.

Proof. Note first that the solutions of (3.2) are solutions of

$$
\begin{aligned}
u(x, t)= & u\left(h^{-1}\left(h(x) e^{-(t-s)}\right), s\right) \exp \left(-\int_{0}^{t-s} g^{\prime}\left(h^{-1}\left(h(x) e^{-\xi}\right)\right) d \xi\right) \\
& +\int_{s}^{t} f\left(u\left(\alpha h^{-1}\left(h(x) e^{-(t-\sigma)}\right), \sigma-\tau\right)\right) \\
& \quad \times \exp \left(-\int_{0}^{t-\sigma} g^{\prime}\left(h^{-1}\left(h(x) e^{-\xi}\right)\right) d \xi\right) d \sigma, \quad 0 \leq x \leq 1,0 \leq s \leq t .
\end{aligned}
$$

Set

$$
t_{0}=0, \quad t_{n+1}=t_{n}+\log \frac{h\left(b \alpha^{-(n+1)}\right)}{h\left(b \alpha^{-n}\right)}+\tau=-\log \frac{h(b)}{h\left(b \alpha^{-(n+1)}\right)}+(n+1) \tau .
$$

We prove by induction that if $b \alpha^{-n} \leq 1$, then

$$
u_{1}(x, t)=u_{2}(x, t) \text { for } x \in\left[0, b \alpha^{-n}\right], t \geq t_{n}-\tau \text {. }
$$

It is true if $n=0$. Suppose true for $n$. If $t \geq t_{n}$,

$$
\begin{aligned}
u_{i}(x, t)= & u_{i}\left(h^{-1}\left(h(x) e^{-\left(t-t_{n}\right)}\right), t_{n}\right) \exp \left(-\int_{0}^{t-t_{n}} g^{\prime}\left(h^{-1}\left(h(x) e^{-\xi}\right)\right) d \xi\right) \\
& +\int_{t_{n}}^{t} f\left(u_{i}\left(\alpha h^{-1}\left(h(x) e^{-(t-\sigma)}\right), \sigma-\tau\right)\right) \\
& \times \exp \left(-\int_{0}^{t-\sigma} g^{\prime}\left(h^{-1}\left(h(x) e^{-\xi}\right)\right) d \xi\right) d \sigma
\end{aligned}
$$


where $i=1,2$. But $h$ and $h^{-1}$ are increasing. Take $x \in\left[0, b \alpha^{-(n+1)}\right], t \geq t_{n+1}-\tau$. Then

$$
\begin{gathered}
h^{-1}\left(h(x) e^{-\left(t-t_{n}\right)}\right) \leq h^{-1}\left(h\left(b \alpha^{-(n+1)}\right) \frac{h\left(b \alpha^{-n}\right)}{h\left(b \alpha^{-(n+1)}\right)}\right)=b \alpha^{-n}, \\
\alpha h^{-1}\left(h(x) e^{-(t-\sigma)}\right) \leq \alpha x \leq b \alpha^{-n},
\end{gathered}
$$

and $\sigma \geq t_{n}$ implies $\sigma-\tau \geq t_{n}-\tau$. Thus $u_{1}(x, t)=u_{2}(x, t)$ for $x \in\left[0, b \alpha^{-(n+1)}\right]$ and $t \geq t_{n+1}-\tau$.

Now suppose that $b \alpha^{-N} \leq 1<b \alpha^{-(N+1)}$. Take $x$ in $[0,1]$. If $\sigma \geq t_{N}$, then

$$
\alpha h^{-1}\left(h(x) e^{-(t-\sigma)}\right) \leq \alpha x \leq b \alpha^{-N},
$$

and if $t \geq t_{N}-\log h\left(b \alpha^{-N}\right)=-\log h(b)+N \tau$,

$$
h^{-1}\left(e^{-\left(t-t_{N}\right)} h(x)\right) \leq h^{-1}\left(h\left(b \alpha^{-N}\right)\right)=b \alpha^{-N} .
$$

So if $x \in[0,1]$ and $t \geq-\log h(b)+N \tau$, then $u_{1}(x, t)=u_{2}(x, t)$. But $N \leq$ $\log b / \log \alpha$, and the result follows.

We now look at the existence of solutions in the case $\tau=0$. If $\tau=0$ and $f$ is only continuous, then solutions may not always exist, as we showed in [2] for the particular cases $g(x)=x$ and $f(x)=\mu x(1-x)$. So consider the case where $f$ is Lipschitz continuous.

Proposition 3.2. Let $\tau=0$, let $\varphi \in X$, and let $f$ be Lipschitz continuous with constant $L$. Then the iterates $u_{n}(x, t)$, defined by

$$
\begin{aligned}
u_{0}(x, t)= & \phi\left(h^{-1}\left(h(x) e^{-t}\right)\right) \exp \left(-\int_{0}^{t} g^{\prime}\left(h^{-1}\left(h(x) e^{-\xi}\right)\right) d \xi\right) \\
u_{n}(x, t)= & \phi\left(h^{-1}\left(h(x) e^{-t}\right)\right) \exp \left(-\int_{0}^{t} g^{\prime}\left(h^{-1}\left(h(x) e^{-\xi}\right)\right) d \xi\right) \\
& +\int_{0}^{t} f\left(u_{n-1}\left(\alpha h^{-1}\left(h(x) e^{-(t-\sigma)}\right), \sigma\right)\right) \\
& \times \exp \left(-\int_{0}^{t-\sigma} g^{\prime}\left(h^{-1}\left(h(x) e^{-\xi}\right)\right) d \xi\right) d \sigma
\end{aligned}
$$

converge, uniformly on compact subsets of $[0,1] \times[0, \infty)$, to the unique continuous solution of (3.2).

If the solution with initial datum $\varphi \in X$ is denoted by $u^{\varphi}(x, t)$, then

$$
\left|u^{\varphi}(\cdot, t)-u^{\psi}(\cdot, t)\right|_{\infty} \leq e^{(L-I) t}|\varphi-\psi|_{\infty}, \quad \varphi, \psi \in X,
$$

where $I=\inf _{0 \leq x \leq 1} g^{\prime}(x)$.

Proof. The convergence of the iterates is proved using standard techniques. 
To prove (3.13), we note that from (3.2), for $x \in[0,1]$,

$$
\left|u^{\varphi}(x, t)-u^{\psi}(x, t)\right| \leq|\varphi-\psi|_{\infty} e^{-I t}+\int_{0}^{t} L\left|u^{\varphi}(x, s)-u^{\psi}(x, s)\right| e^{-I(t-s)} d s
$$

and the result follows immediately using Gronwall's inequality.

If $f$ is locally Lipschitz continuous, uniqueness can be proved using Gronwall's inequality and we have the following lemma.

LEMMA 3.3. Let $\tau=0$ and let $\varphi \in X$. If $f$ is locally Lipschitz continuous, then there is at most one solution of (3.2).

Also we have the following lemma.

LEMMA 3.4. Let $\tau=0$ and let $\varphi \in X$. If, for some $\varphi \in C[0,1]$, there exists $b, 0<b \leq 1$, such that (3.2) has a solution for $x \in[0, b]$, then (3.2) also has $a$ solution for $x \in[0,1]$.

Proof. Take $x \in\left[0, b \alpha^{-1}\right]$. Then $\alpha h^{-1}\left(h(x) e^{-(t-\sigma)}\right) \in[0, b]$ and so $u(x, t)$ is defined for $x \in\left[0, b \alpha^{-1}\right]$. Continue by induction.

This leads to the following proposition. Denote by $X_{0}$ the space $\{\varphi \in X$, $\varphi(0)=0\}$ and by $X_{0}^{+}$the set $\left\{\varphi \in X_{0}, \varphi \geq 0\right\}$. If $0<b<1$ and $\varphi \in C[0, b]$, we set $|\varphi|_{b}=\sup _{0 \leq x \leq b}|\varphi(x)|$ and if $\varphi \in C([0, b] \times[-\tau, 0])$, where $\tau>0$, we set $\|\varphi\|_{b}=\sup _{0 \leq x \leq b,-\tau \leq t \leq 0}|\varphi(x, t)|$.

Proposition 3.5. Let $\tau=0$.

(a) If there exists $\eta>0$ such that if $|\varphi|_{\infty}<\eta$ and $\varphi \in X_{0}$ then $u^{\varphi}(x, t)$ exists, then in fact (3.2) has a solution for all $\varphi \in X_{0}$.

(b) If there exists $\eta>0$ such that if $0 \leq \varphi<\eta$ and $\varphi \in X_{0}$ then $u^{\varphi}(x, t)$ exists, then in fact (3.2) has a solution for all $\varphi \in X_{0}^{+}$.

Proof. To prove (a), given $\varphi \in X_{0}$, there exists $b$ such that $|\varphi|_{b}<\eta$. Define $\psi \in X_{0}$ by

$$
\psi(x)= \begin{cases}\varphi(x) & \text { if } x \in[0, b], \\ \varphi(b) & \text { if } x \in[b, 1] .\end{cases}
$$

Then $|\psi|_{\infty}<\eta$ and thus $u^{\psi}(x, t)$ exists. So $u^{\varphi}(x, t)$ exists for $x \in[0, b]$ as $\varphi(x)=\psi(x)$ for $x \in[0, b]$. The result now follows from Lemma 3.4.

The proof of (b) is similar.

Finally, we have the following existence result for $f$ locally Lipschitz continuous.

THEOREM 3.6. Let $\tau=0$. Suppose that $g$ satisfies $\left(H_{g}\right)$ and also $g^{\prime}(x)>0$, for $0 \leq x \leq 1$. Suppose that $f \in C^{1}(\mathbb{R})$ and $f(0)=0$. Set $I=\inf _{0 \leq x \leq 1} g^{\prime}(x)$. Suppose that either 
(a) there is a $\delta>0$ such that $\left|f^{\prime}(x)\right| \leq I$ for $|x| \leq \delta$ and the initial datum $\varphi \in X$ is such that, for some $b>0,|\varphi|_{b} \leq \delta$, or

(b) there is a $\delta>0$ such that $0 \leq f^{\prime}(x) \leq I$ for $0 \leq x \leq \delta$ and the initial datum $\varphi \in X$ is such that, for some $b>0,0 \leq \varphi(x) \leq \delta$ for $0 \leq x \leq b$.

Then the iterates $u_{n}(x, t)$ defined in (3.12) converge uniformly on compact subsets of $[0, b] \times[0, \infty)$ to a function $u(x, t)$ which satisfies (3.2) for $x \in[0, b]$.

Thus, for such $\varphi$, (3.2) has a unique solution for $x \in[0,1]$. In particular, under condition (a) there is a solution for all $\varphi \in X_{0}$ and under condition (b) there is a solution for all $\varphi \in X_{0}^{+}$.

Proof. We prove (a). Let $\mu=\sup _{|x| \leq \delta}\left(\left|f^{\prime}(x)\right| / I\right)$. We have $f(k)-f(0)=$ $f^{\prime}(\xi) k$ and so $|f(k)| \leq \mu \delta I$ if $|k|<\delta$. If $|\varphi|_{b} \leq \delta$ and $\left|u_{n-1}(\cdot, t)\right|_{b} \leq \delta$, then

$$
\left|u_{n}(\cdot, t)\right|_{b} \leq \delta e^{-I t}+\int_{0}^{t} \mu I \delta e^{-I(t-s)} d s \leq \delta .
$$

Hence by induction $\left|u_{n}(\cdot, t)\right|_{b} \leq \delta$ for all $n$. The convergence of the $u_{n}(x, t)$ uniformly for $x \in[0, b]$ and $t$ on compact subsets of $[0, \infty)$ now follows by standard iterative techniques.

That there is a solution of (3.2) for $x \in[0,1]$ follows from Lemma 3.4 and that there is a solution for any $\varphi \in X_{0}$ follows from Proposition 3.5(a).

The proof of (b) is similar.

Note that what we need in the proof of Theorem 3.6 is that $f^{\prime}(x)$ exists in $[-\gamma, \gamma]$ for a $\gamma>0$.

4. Invariance and asymptotic behavior. In this section, we study invariance and asymptotic behavior of solutions. In Theorem 4.1 we provide sufficient conditions on the relationship between the population growth and maturation processes to guarantee the extinction of the population for a class of initial data.

THEOREM 4.1. Let $\tau>0$. Suppose that $g$ satisfies $\left(H_{g}\right)$ and also $g^{\prime}(x)>0$, for $0 \leq x \leq 1$. Set $I=\inf _{0 \leq x \leq 1} g^{\prime}(x)$. Suppose that $f \in C^{1}(\mathbb{R})$ and $f(0)=0$. Suppose that either

(a) there is a $\delta>0$ such that $\left|f^{\prime}(x)\right|<I$ for $|x| \leq \delta$ and the initial datum $\varphi$ is such that, for some $b>0,\|\varphi\|_{b} \leq \eta \leq \delta$, or

(b) there is a $\delta>0$ such that $0 \leq f^{\prime}(x)<I$ for $0 \leq x \leq \delta$ and the initial datum $\varphi \in Y$ is such that, for some $b>0,0 \leq \varphi(x, t) \leq \eta \leq \delta$, for $0 \leq x \leq b$, $-\tau \leq t \leq 0$.

Then invariance of the initial datum bounds holds,

$$
|u(\cdot, t)|_{b} \leq \eta, t \geq 0,
$$

and in case (b),

$$
0 \leq u(x, t) \leq \eta, 0 \leq x \leq b, t \geq 0 .
$$


Also $u(\cdot, t) \rightarrow 0$ exponentially in $Y$ as $t \rightarrow \infty$. The results hold with obvious modifications if $\tau=0$.

Proof. Take $\tau>0$ first and look at case (a).

Set $\mu=\sup _{|x| \leq \delta}\left(\left|f^{\prime}(x)\right| / I\right)$. We have $f(h)-f(0)=f^{\prime}(\xi) h$, so $|f(h)| \leq \mu \eta I$ if $|h| \leq \eta$.

Take $t \in[0, \tau], x \in[0, b]$, and $\varphi$ such that $\|\varphi\|_{b} \leq \eta$, then

$$
|u(\cdot, t)|_{b} \leq \eta e^{-I t}+\int_{0}^{t} \mu \eta I e^{-I(t-s)} d s \leq \eta\left(e^{-I t}(1-\mu)+\mu\right) .
$$

Thus in particular $|u(\cdot, t)|_{b} \leq \eta$ for $t \in[0, \tau]$, and, by induction, $|u(\cdot, t)|_{b} \leq \eta$ for all $t \geq 0$, and so (4.3) holds for all $t \geq 0$.

Now take $\mu<\lambda<1$ and set $e^{-I(T-\tau)}(1-\mu)=\lambda-\mu$, so

$$
|u(\cdot, t)|_{b} \leq \eta \lambda \text { for } t \geq T-\tau \text {. }
$$

Thus we can prove, by induction, as in [2, Theorem 6.1], that

$$
|u(\cdot, t)|_{b} \leq \eta \lambda^{n} \quad \text { for } t \geq n T-\tau,
$$

and so

$$
|u(\cdot, t)|_{\infty} \leq \eta \lambda^{n} \quad \text { for } t \geq-\log h(b)+\frac{\log b}{\log \alpha} \tau+n T-\tau,
$$

and we have exponential decay.

Now look at the case (b) and $\tau>0$.

Set $\mu=\sup _{0 \leq x \leq \delta}\left(f^{\prime}(x) / I\right)$ and note that, as above, if $0 \leq h \leq \delta, f(h)=$ $f^{\prime}(\xi) h$, and so $0 \leq f(h) \leq \eta I \mu$ if $0 \leq h \leq \eta$.

Take $t \in[0, \tau], x \in[0, b]$, so

$$
u(\cdot, t) \leq \eta e^{-I t}+\int_{0}^{t} \mu \eta I e^{-I(t-s)} d s \leq \eta\left(e^{-I t}(1-\mu)+\mu\right),
$$

also $u(x, t) \geq 0$. Thus, in particular, $0 \leq u(x, t) \leq \eta$ for $x \in[0, b]$ and $t \in[0, \tau]$, and hence, by induction, $0 \leq u(x, t) \leq \eta$ for $x \in[0, b]$ and $t \geq 0$.

Now proceed as before using (4.7) instead of (4.3).

We now look at the case $\tau=0$. Consider $u_{n}(x, t)$ as defined by (3.12). It is easily seen by induction on $n$ that in case (a)

$$
\text { if }|\varphi|_{b} \leq \eta \text {, then }\left|u_{n}(\cdot, t)\right|_{b} \leq \eta \text { for } t \geq 0 \text {, }
$$

and in case (b)

$$
\text { if } 0 \leq \varphi(x) \leq \eta \text {, then } 0 \leq u_{n}(x, t) \leq \eta \text { for } x \in[0, b], t \geq 0 \text {. }
$$

Thus, letting $n \rightarrow \infty$ in (4.8) and (4.9) we have the invariance results. 
Now in case (a), from (4.8), we have

$$
\left|u_{n}(\cdot, t)\right|_{b} \leq \eta e^{-I t}+\int_{0}^{t} \mu \eta I e^{-I(t-s)} d s=\eta\left(e^{-I t}(1-\mu)+\mu\right) \quad \text { for } t \geq 0,
$$

and so $\left|u_{n}(\cdot, t)\right|_{b} \leq \eta \lambda$ for $t \geq T$, and, as above, by induction, $\left|u_{n}(\cdot, t)\right|_{b} \leq \eta \lambda^{N}$ for $t \geq N T$. It follows that

$$
|u(\cdot, t)|_{b} \leq \eta \lambda^{N} \text { for } t \geq N T
$$

Hence we have exponential decay. Similarly in case (b).

A similar result was proved in [2] for the particular case $g(x)=x$ and $f(x)=$ $\mu x(1-x)$.

5. Instability. In this section, we study the instability of solutions. We denote by $u^{\phi}$ the solution of (3.2) with initial datum $\phi$; note that when $\tau>0$, $\phi \in Y$ and when $\tau=0, \phi \in X$. If $\tau>0$ and $\phi \in Y$, we say that $u^{\phi}$ is stable if given $\varepsilon>0$, there exists a $\delta>0$ such that if $\psi \in Y$ and $\|\phi-\psi\|<\delta$, then $\left|u^{\phi}(\cdot, t)-u^{\psi}(\cdot, t)\right|_{\infty}<\varepsilon$ for all $t$. If $\tau=0$ and $\phi \in X$, we say that $u^{\phi}$ is stable if given $\varepsilon>0$, there exists a $\delta>0$ such that if $\psi \in X$ and $|\phi-\psi|_{\infty}<\delta$, then $\left|u^{\phi}(\cdot, t)-u^{\psi}(\cdot, t)\right|_{\infty}<\varepsilon$ for all $t$. We say that $u^{\phi}$ is unstable if it is not stable.

The following hypothesis, which assumes that the population does not extinguish for at least one initial condition, is sufficient in certain cases to guarantee the instability of all solutions for a certain class of initial conditions. The interpretation of this result is that the population behavior is abnormal either in its inability to stabilize to a normal level or its inability to survive at all.

HyPOTHESIS 1 . There exists a solution $u^{\phi}$ such that $\left|u^{\phi}(\cdot, t)\right|_{\infty}$ does not converge to 0 as $t$ tends to $\infty$.

We denote by $Y_{0}$ the space $\{\varphi \in Y, \varphi(0, t)=0$ for $t \in[-\tau, 0]\}$. We prove that if this condition is satisfied for a $\phi$ in $Y_{0}$, then for all $\phi$ in $Y_{0}, u^{\phi}$ is unstable. As we will see in the proof, this is a consequence of the uniqueness result proved in Proposition 3.1, that is, that if initial data coincide for $x \in[0, b]$, where $0<b<1$, then eventually solutions coincide, and that if the functions $\phi_{1}$ and $\phi_{2}$ belong to $Y_{0}$, then, if $b$ is small, $\left|\phi_{1}(t, x)-\phi_{2}(t, x)\right|$ is small for $x \in[0, b]$.

We first consider the case $\tau>0$.

Proposition 5.1. Let $\tau>0$. Suppose that Hypothesis 1 holds for a solution with initial datum $\phi_{0} \in Y_{0}$. Then for every $\phi \in Y_{0}, u^{\phi}$ is unstable.

Proof. If Hypothesis 1 holds, there exists $\varepsilon>0$ and $\sigma_{n} \rightarrow \infty$ such that $\left|u^{\phi_{0}}\left(\cdot, \sigma_{n}\right)\right|_{\infty}>\varepsilon$ for all $n$.

Suppose that $\phi \in Y_{0}$ is stable. So there exists $\delta>0$ such that if $\|\phi-\psi\|<\delta$, then $\left|u^{\phi}(\cdot, t)-u^{\psi}(\cdot, t)\right|_{\infty}<\varepsilon / 2$ for all $t \geq 0$. 
Choose $b_{1}>0$ and $\phi_{1} \in Y_{0}$ such that $\phi_{1}(x, t)=0$ in $\left[0, b_{1}\right] \times[-\tau, 0]$ and $\left\|\phi_{1}-\phi\right\|<\delta$ so that $\left|u^{\phi_{1}}(\cdot, t)-u^{\phi}(\cdot, t)\right|_{\infty}<\varepsilon / 2$ for all $t \geq 0$.

Also we can choose $b_{2}>0$ and $\phi_{2} \in Y_{0}$ such that $\phi_{2}(x, t)=\phi_{0}(x, t)$ in $\left[0, b_{2}\right] \times[\tau, 0]$ and $\left\|\phi_{2}-\phi\right\|<\delta$ so that $\left|u^{\phi_{2}}(\cdot, t)-u^{\phi}(\cdot, t)\right|_{\infty}<\varepsilon / 2$ for all $t \geq 0$.

By Proposition 3.1 there exist $t_{1}$ and $t_{2}$ such that $u^{\phi_{1}}(x, t)=0$ if $t>t_{1}$ and $u^{\phi_{2}}(x, t)=u^{\phi_{0}}(x, t)$ if $t>t_{2}$.

Hence if $t>\max \left\{t_{1}, t_{2}\right\}$, then $\left|u^{\phi}(\cdot, t)\right|_{\infty}<\varepsilon / 2$ and $\left|u^{\phi_{0}}(\cdot, t)-u^{\phi}(\cdot, t)\right|_{\infty}<$ $\varepsilon / 2$. So if $t>\max \left\{t_{1}, t_{2}\right\},\left|u^{\phi_{0}}(\cdot, t)\right|_{\infty}<\varepsilon$, a contradiction.

We now prove that Hypothesis 1 is satisfied if $g(x)=x$.

THEOREM 5.2. Let $\tau>0$. Consider the equation

$$
\begin{gathered}
u(x, t)=e^{-t} \phi\left(e^{-t} x, 0\right)+\int_{0}^{t} e^{-(t-s)} f\left(u\left(\alpha x e^{-(t-s)}, s-\tau\right)\right) d s, \quad t \geq 0 \\
u(x, t)=\phi(x, t), \quad x \in[0,1], t \in[-\tau, 0]
\end{gathered}
$$

Suppose that $f(0)=0$ and $f^{\prime}(0)>1$. Then there exists $\phi_{0} \in Y_{0}$ such that $u^{\phi_{0}}(\cdot, t)$ does not converge in $X$ to 0 as $t \rightarrow \infty$, and so all $u^{\phi}$ with initial datum $\phi \in Y_{0}$ are unstable.

Proof. Let $\varepsilon>0$ be such that $f^{\prime}(0)-\varepsilon>1 ; f(y)=f^{\prime}(0) y+o(y)$, where $o(y) / y \rightarrow 0$ as $y \rightarrow 0$, and so there exists $\delta>0$ such that if $0 \leq y \leq \delta, o(y)+$ $\varepsilon y \geq 0$. Set $\mu=f^{\prime}(0)-\varepsilon$, so $f(y)=\mu y+h(y)$, where $h(y)=o(y)+\varepsilon y$ is such that

$$
h(y) \geq 0 \text { if } 0 \leq y \leq \delta
$$

Choose

$$
\phi(x, t)=\delta x^{r} e^{s t}, \quad 0 \leq x \leq 1,-\tau \leq t \leq 0,
$$

where $r>0$ and $s>0$ are such that $r+s+1=\mu \alpha^{r} e^{-s \tau}$. Note that $u_{\mu}(x, t)=$ $\delta x^{r} e^{s t}$ is the solution of the equation

$$
\begin{gathered}
u_{t}(x, t)+(x u(x, t))_{x}=\mu u(\alpha x, t-\tau), \quad t>0, \\
u(x, t)=\delta x^{r} e^{s t}, \quad 0 \leq x \leq 1,-\tau \leq t \leq 0 .
\end{gathered}
$$

Let $u(x, t)$ be the solution of (5.1) with $\phi(x, t)=\delta x^{r} e^{s t}$. Suppose, for contradiction, that $u(\cdot, t) \rightarrow 0$ as $t \rightarrow \infty$. Thus there exists $x_{1}, 0<x_{1} \leq 1$, such that

$$
|u(x, t)| \leq \delta \text { for } 0 \leq x \leq x_{1}, t \geq-\tau \text {. }
$$


Set

$$
v(x, t)=u(x, t)-u_{\mu}(x, t)
$$

we have

$$
\begin{aligned}
u(x, t)= & e^{-t} \phi\left(x e^{-t}, 0\right)+\int_{0}^{t} e^{-(t-\sigma)} f\left(u\left(e^{-(t-\sigma)} \alpha x, \sigma-\tau\right)\right) d \sigma \\
= & e^{-t} \phi\left(x e^{-t}, 0\right)+\int_{0}^{t} e^{-(t-\sigma)} \mu u_{\mu}\left(e^{-(t-\sigma)} \alpha x, \sigma-\tau\right) d \sigma \\
& +\int_{0}^{t} e^{-(t-\sigma)} \mu v\left(e^{-(t-\sigma)} \alpha x, \sigma-\tau\right) d \sigma \\
& +\int_{0}^{t} e^{-(t-\sigma)} h\left(u\left(e^{-(t-\sigma)} \alpha x, \sigma-\tau\right)\right) d \sigma .
\end{aligned}
$$

But $e^{-t} \phi\left(x e^{-t}, 0\right)+\int_{0}^{t} e^{-(t-\sigma)} \mu u_{\mu}\left(e^{-(t-\sigma)} \alpha x, \sigma-\tau\right) d \sigma=u_{\mu}(x, t)$, so

$$
\begin{aligned}
v(x, t)= & \int_{0}^{t} e^{-(t-\sigma)} \mu v\left(e^{-(t-\sigma)} \alpha x, \sigma-\tau\right) d \sigma \\
& +\int_{0}^{t} e^{-(t-\sigma)} h\left(u\left(e^{-(t-\sigma)} \alpha x, \sigma-\tau\right)\right) d \sigma .
\end{aligned}
$$

Hence for $0 \leq t \leq \tau$,

$$
v(x, t)=0+\int_{0}^{t} e^{-(t-\sigma)} h\left(\phi\left(e^{-(t-\sigma)} \alpha x, \sigma-\tau\right)\right) d \sigma \geq 0
$$

because $0 \leq \phi(x, t) \leq \delta$, for $0 \leq x \leq 1,-\tau \leq t \leq 0$, and so

$$
u(x, t) \geq u_{\mu}(x, t) \geq 0 \text { for } 0 \leq x \leq 1,0 \leq t \leq \tau .
$$

It follows, in particular, that

$$
0 \leq u(x, t) \leq \delta \text { for } 0 \leq x \leq x_{1}, 0 \leq t \leq \tau,
$$

and so

$$
h(u(x, t)) \geq 0 \text { for } 0 \leq x \leq x_{1}, 0 \leq t \leq \tau,
$$

by (5.2). Hence, by (5.8), $v(x, y) \geq 0$, for $0 \leq x \leq x_{1}$ and $0 \leq t \leq 2 \tau$, and, by induction,

$$
v(x, y) \geq 0 \text { for } 0 \leq x \leq x_{1}, t \geq 0 .
$$

Thus for $0<x \leq x_{1}$,

$$
u(x, t) \geq u_{\mu}(x, t)=\delta x^{r} e^{s t} \longrightarrow \infty \quad \text { as } t \longrightarrow \infty,
$$

giving a contradiction. 
A similar argument to that given in the proof of Proposition 5.1 holds for $\tau=0$ if there is existence of solutions for all initial data in $X_{0}$. Proposition 3.5 gives a sufficient condition for existence of solutions for all such initial data. So for the case $\tau=0$ we have the instability result below.

Proposition 5.3. Let $\tau=0$. Suppose that there is an $\eta$ such that there are solutions of (3.2) for all $\varphi \in X_{0}$ such that $|\varphi|_{\infty}<\eta$. Suppose that Hypothesis 1 holds for a solution with initial data in $X_{0}$. Then for every $\phi \in X_{0}, u^{\phi}$ is unstable.

Finally we have the analogue of Theorem 5.2 for the case $\tau=0$.

THEOREM 5.4. Let $\tau=0$. Consider the equation

$$
\begin{gathered}
u(x, t)=e^{-t} \varphi\left(e^{-t} x\right)+\int_{0}^{t} e^{-(t-s)} f\left(u\left(\alpha x e^{-(t-s)}, s\right)\right) d s, \quad t>0, x \in[0,1], \\
u(x, 0)=\varphi(x), \quad x \in[0,1] .
\end{gathered}
$$

Assume that there is an $\eta>0$ such that if $\varphi \in X_{0}$ and $|\varphi|_{\infty}<\eta$, then there exists a solution $u^{\varphi}(x, t)$ and if $\varphi \geq 0$, then $u^{\varphi}(x, t) \geq 0$ and $u^{\varphi}(0, t)=0$ for all $t \geq 0$. Suppose that $f(0)=0$ and $f^{\prime}(0)>1$. Then there exists $\phi_{0} \in X_{0}$ such that $u^{\phi_{0}}(\cdot, t)$ does not converge in $X$ to 0 as $t \rightarrow \infty$, and so all $u^{\phi}$ with initial datum $\phi \in X_{0}$ are unstable.

Proof. We use the same notation as in the proof of Theorem 5.2 but take $\delta \leq \eta$. Denote by $U_{\mu}(t) \phi$ the semigroup of the solutions of the equation

$$
\begin{gathered}
u_{t}(x, t)+(x u(x, t))_{x}=\mu u(\alpha x, t), \quad t>0, \\
u(x, 0)=\phi(x) .
\end{gathered}
$$

In Section 2 (and, in fact, also in [2]) we prove that the semigroup generated in $C[0,1]$ by the operator $-(x u(x))^{\prime}$ with domain

$$
\left\{u \in C[0,1], u \text { is differentiable on }(0,1], u^{\prime} \in C(0,1], \lim _{x \rightarrow 0} x u^{\prime}(x)=0\right\}
$$

is $(V(t) \phi)(x)=e^{-t} \phi\left(e^{-t} x\right)$. Also the semigroup generated in $C[0,1]$ by the continuous operator $(H \phi)(x)=\mu \phi(\alpha x)$ is

$$
(W(t) \phi)(x)=\sum_{n=1}^{\infty} \frac{t^{n}}{n !}\left(H^{n} \phi\right)(x)=\sum_{n=1}^{\infty} \frac{(\mu t)^{n}}{n !} \phi\left(\alpha^{n} x\right) .
$$

Note that $V(t) W(t)=W(t) V(t)$. It follows that $U_{\mu}(t)=W(t) T(t)$, that is,

$$
\left(U_{\mu}(t) \phi\right)(x)=\sum_{n=0}^{\infty} \frac{(\mu t)^{n}}{n !} e^{-t} \phi\left(\alpha^{n} e^{-t} x\right),
$$


and so, if $\phi \geq 0$, then $U_{\mu}(t) \phi \geq 0$. Now take $r>0, s>0$ such that $r+s+1=$ $\mu \alpha^{r}$. Choose $\phi=\delta x^{r}$, so that $U_{\mu}(t) \phi=\delta x^{r} e^{s t}$. Let $u(x, t)$ be the solution of (5.15) with initial datum $\phi(x)=\delta x^{r}$. Suppose, for contradiction, that $u(\cdot, t) \rightarrow$ 0 in $X$ as $t \rightarrow \infty$. As $f(y)=\mu y+h(y)$, we have

$$
u(x, t)=U_{\mu}(t) \phi(x)+\int_{0}^{t} U_{\mu}(t-s) h(u(\alpha x, \sigma)) d \sigma
$$

Since $u(\cdot, t) \rightarrow 0$ in $X$ as $t \rightarrow \infty$ and $u(0, t)=0$, for all $t \geq 0$, there exists $x_{1}, 0<x_{1} \leq 1$, such that $0 \leq u(x, t)<\delta$ for $0 \leq x \leq x_{1}$ and $t \geq 0$. Thus $h(u(\alpha x, \sigma)) \geq 0$, for $0 \leq x \leq x_{1} / \alpha$ and $\sigma \geq 0$, so that

$$
u(x, t) \geq\left(U_{\mu}(t) \phi\right)(x)=\delta x^{r} e^{s t}
$$

for $0 \leq x \leq x_{1} / \alpha$ and $t \geq 0$, yielding a contradiction.

6. Summary and conclusions. The production of blood cells is a complex process that originates from an experimentally inaccessible source of most primitive cells. Through successive divisions, cell lines exhibit an increasing maturity of cell types as individual cells grow and divide. The ultimate behavior of the population is inextricably linked to this behavior of individual cells. The model we have developed is a simplified description of this maturationproliferation process. It consists of a semilinear partial differential equation of transport type, for which we investigate the existence, uniqueness, and asymptotic behavior of solutions. In this model the normal production of blood cells depends on the initial state of the system. If there is not a sufficient supply in the initial state of the most primitive cells, then the population either extinguishes or destabilizes. It is hypothesized that abnormalities of the blood production system, such as aplastic anemia, arise as defects in or shocks to the most primitive precursor cells. The results we have established provide a qualitative conceptualization of this kind of abnormal population process.

ACKNOWLEDGment. The research of the second author was supported in part by MURST grant.

\section{REFERENCES}

[1] M. Adimy and L. Pujo-Menjouet, A singular transport model describing cellular division, C. R. Acad. Sci. Paris Sér. I Math. 332 (2001), no. 12, 1071-1076.

[2] J. Dyson, R. Villella-Bressan, and G. Webb, A singular transport equation modelling a proliferating maturity structured cell population, Canad. Appl. Math. Quart. 4 (1996), no. 1, 65-95.

[3] T. R. Harrison (ed.), Principles of Internal Medicine, 9th ed., McGraw-Hill, New York, 1980.

[4] L. L. Henry, Population Analysis and Models, Edward Arnold, London, 1976.

[5] K. E. Howard, A size structured model of cell dwarfism, Discrete Contin. Dyn. Syst. Ser. B 1 (2001), no. 4, 471-484. 
[6] N. Keyfitz, Introduction to the Mathematics of Population, Addison-Wesley, Massachusetts, 1968.

[7] M. C. Mackey and P. Dörmer, Continuous maturation of proliferating erythroid precursors, Cell and Tissue Kinetics 15 (1982), 381-392.

[8] M. C. Mackey and R. Rudnicki, A new criterion for the global stability of simultaneous cell replication and maturation processes, J. Math. Biol. 38 (1999), no. 3, 195-219.

[9] J. H. Pollard, Mathematical Models for the Growth of Human Populations, Cambridge University Press, Cambridge, 1973.

[10] A. D. Rey and M. C. Mackey, Multistability and boundary layer development in a transport equation with delayed arguments, Canad. Appl. Math. Quart. 1 (1993), no. 1, 61-81.

Janet Dyson: Mansfield College, Oxford, OX1 3TF, UK

E-mail address: janet.dyson@mansfie1d.oxford.ac.uk

Rosanna Villella-Bressan: Dipartimento di Matematica Pura ed Applicata, Universitá di Padova, Padova, Italy

E-mail address: rosannav@math. unipd.it

Glenn F. Webb: Department of Mathematics, Vanderbilt University, Nashville, TN 37235, USA

E-mail address: g1enn.f.webb@vanderbi 1t.edu 


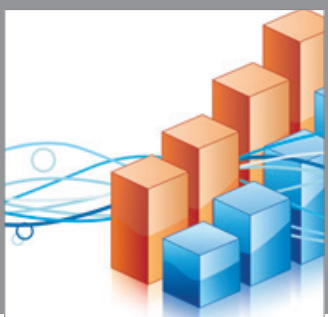

Advances in

Operations Research

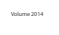

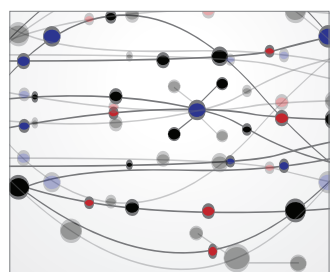

\section{The Scientific} World Journal
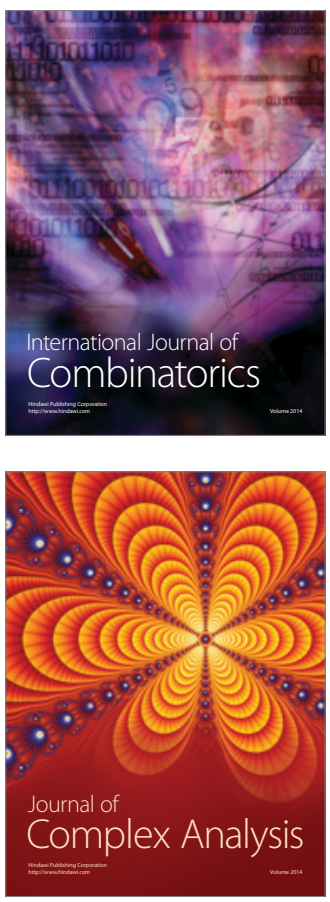

International Journal of

Mathematics and

Mathematical

Sciences
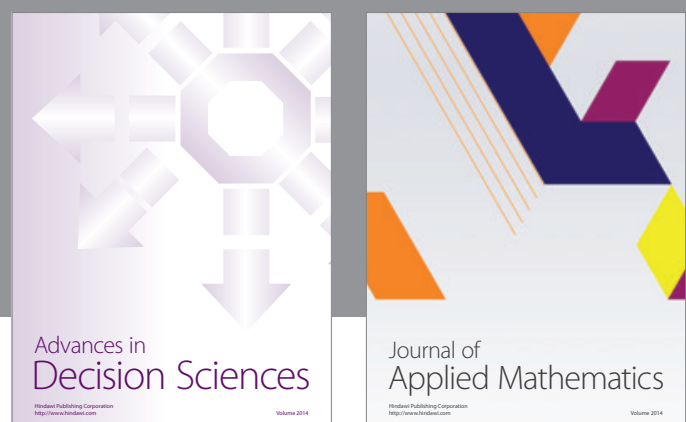

Journal of

Applied Mathematics
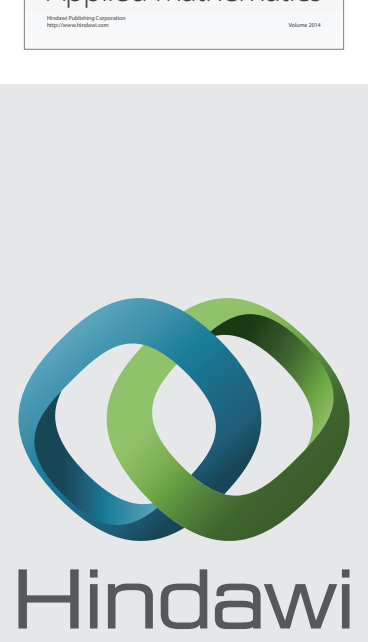

Submit your manuscripts at http://www.hindawi.com
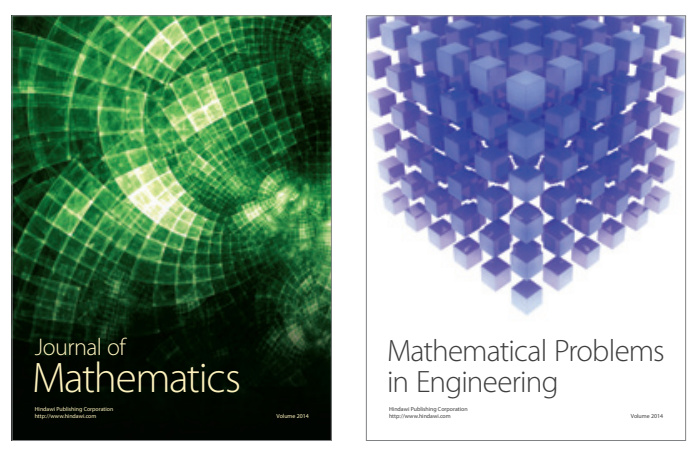

Mathematical Problems in Engineering
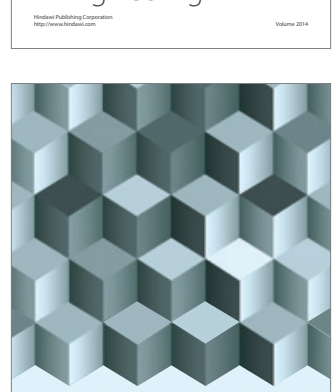

Journal of

Function Spaces
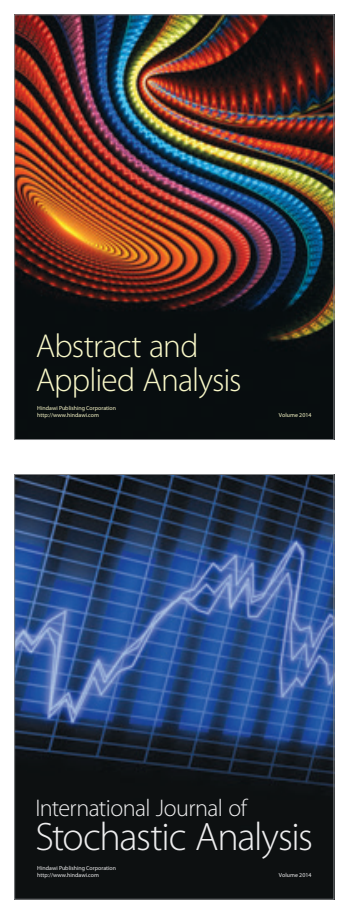

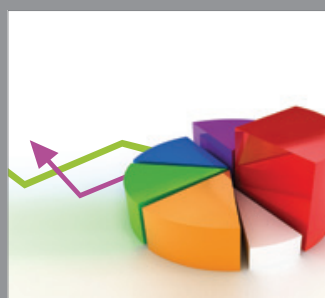

ournal of

Probability and Statistics

Promensencen
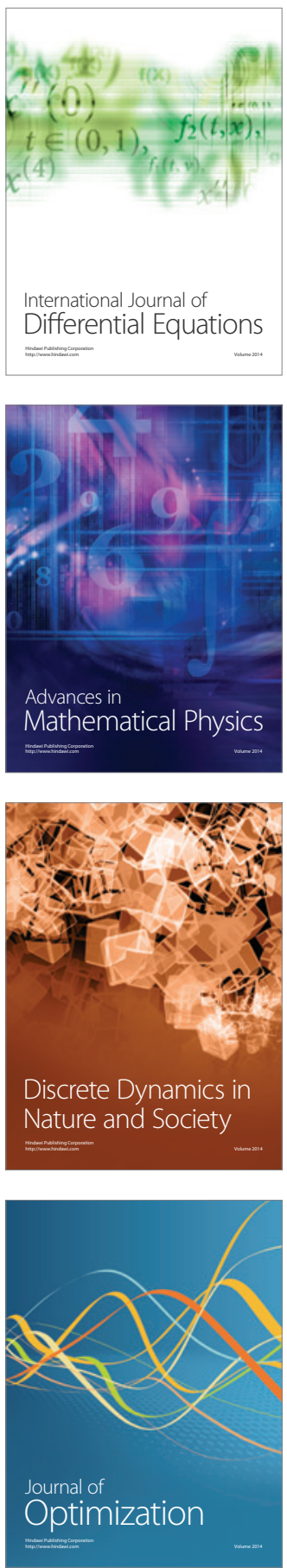